\title{
ADVANCED
}

Supporting Information

for Adv. Mater., DOI: 10.1002/adma.201102199

Continuous Patterning of Nanogratings by Nanochannel-Guided Lithography on Liquid Resists

Jong G. Ok, Hui Joon Park, Moon Kyu Kwak, Carlos A. PinaHernandez, Se Hyun Ahn, and L. Jay Guo * 


\title{
Supporting Information
}

\section{Continuous patterning of nanogratings by NanoChannel-guided Lithography (NCL) on liquid resists}

\author{
Jong G. Ok, ${ }^{1}$ Hui Joon Park, ${ }^{2}$ Moon Kyu Kwak, ${ }^{3}$ Carlos A. Pina-Hernandez, ${ }^{3}$ Se Hyun Ahn, ${ }^{1, \dagger}$ and L. Jay Guo ${ }^{2,3, *}$ \\ Center for Nanophotonics and Spintronics \\ ${ }^{1}$ Department of Mechanical Engineering, ${ }^{2}$ Macromolecular Science and Engineering, ${ }^{3}$ Department of \\ Electrical Engineering and Computer Science, University of Michigan, Ann Arbor, MI 48109 (USA) \\ Current address: Molecular Imprints, Inc., Austin, TX 78758 (USA) \\ *E-mail: guo@umich.edu
}
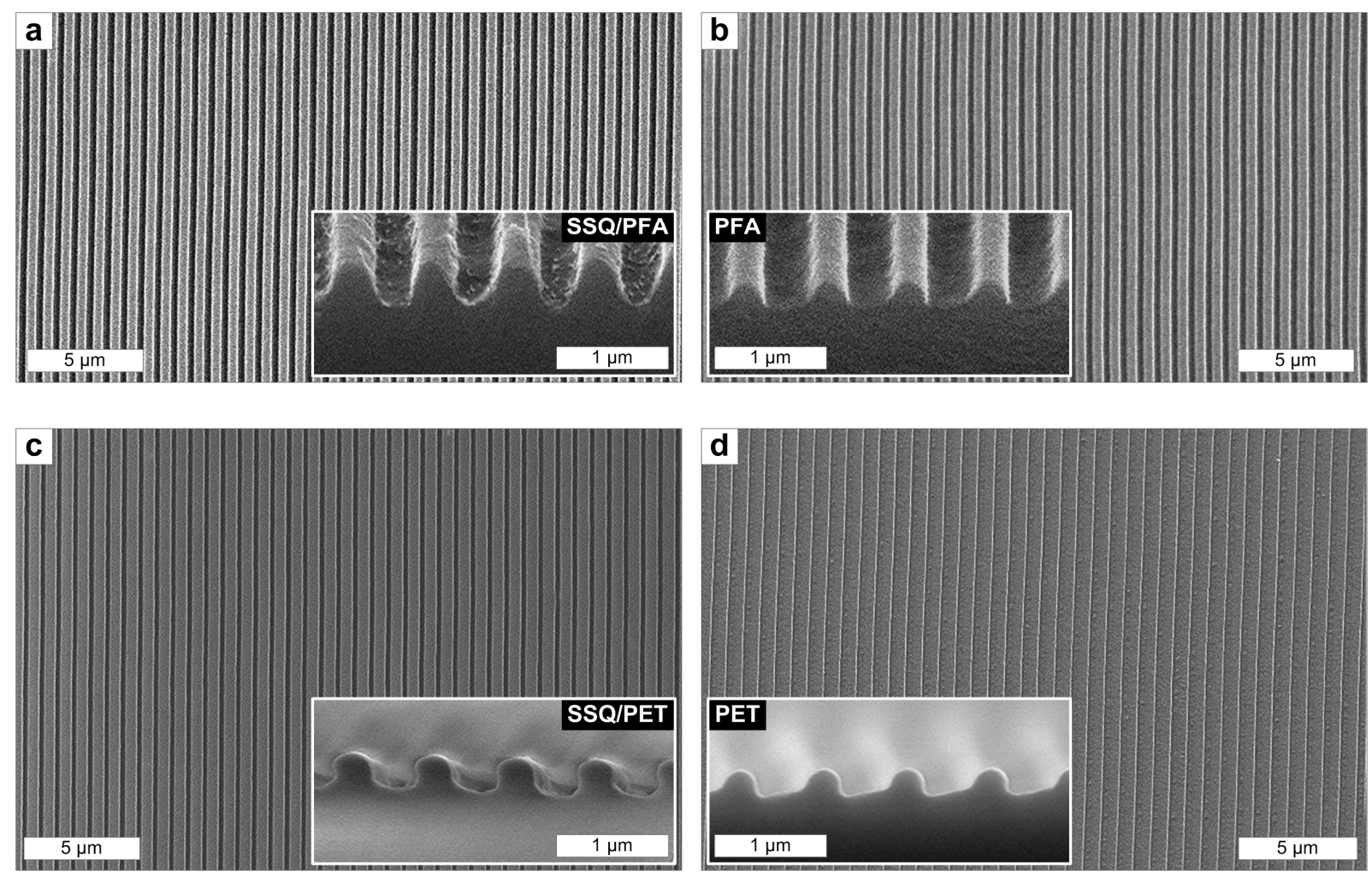

Figure S1. SEM images of $700 \mathrm{~nm}$ period nanogratings formed on the PFA substrates (a) with and (b) without SSQ coating, and formed on the PET substrates (c) with and (d) without SSQ coating. All are processed at $80{ }^{\circ} \mathrm{C}$. The insets are the counterprofiles of each grating structure, evidently showing that the aspect ratio of resulting nanogratings can be significantly enhanced by the use of SSQ layer. These are all consistent with the result obtained in $200 \mathrm{~nm}$ period nanogratings on PFA substrates, as shown in the main text (Figures 1e and 1f). 


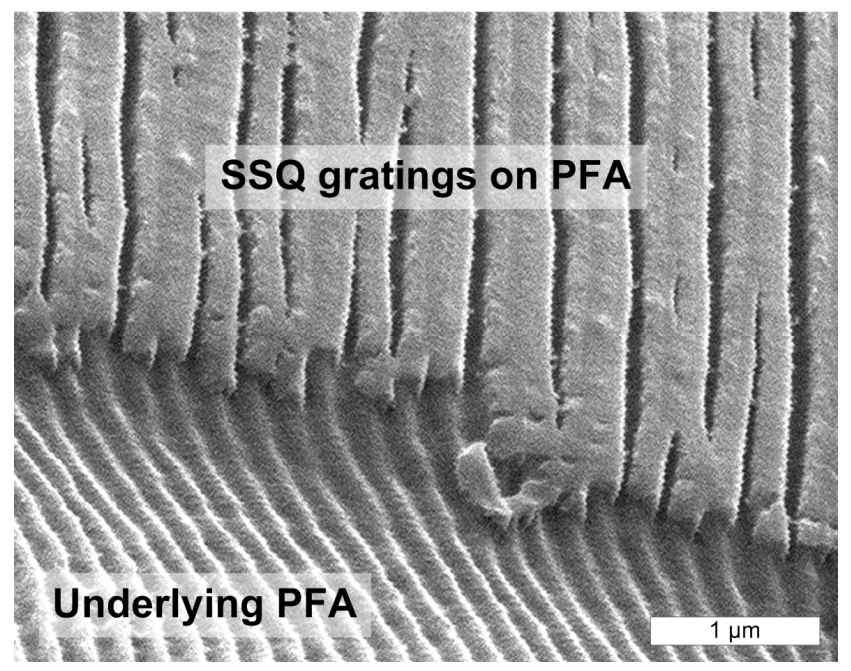

Figure S2. SEM image of the exposed underlying PFA surfaces after the NCL process, showing deformed morphology along the mold transfer direction. The sample was fabricated by applying the 200 $\mathrm{nm}$ period mold on the SSQ-coated PFA substrate at $80{ }^{\circ} \mathrm{C}$, followed by full curing under intense UV light. To make this boundary, the fully cured SSQ/PFA nanograting sample was first vapor-treated with Silquest A-187 at $90^{\circ} \mathrm{C}$ for 5 min. Next, epoxysilicone was partially applied (drop-casting) and UV-cured, then was quickly delaminated by a razor blade. The SSQ/PFA grating at the boundary look somewhat stuck to each other because of capillary infiltration of residual epoxysilicone. 


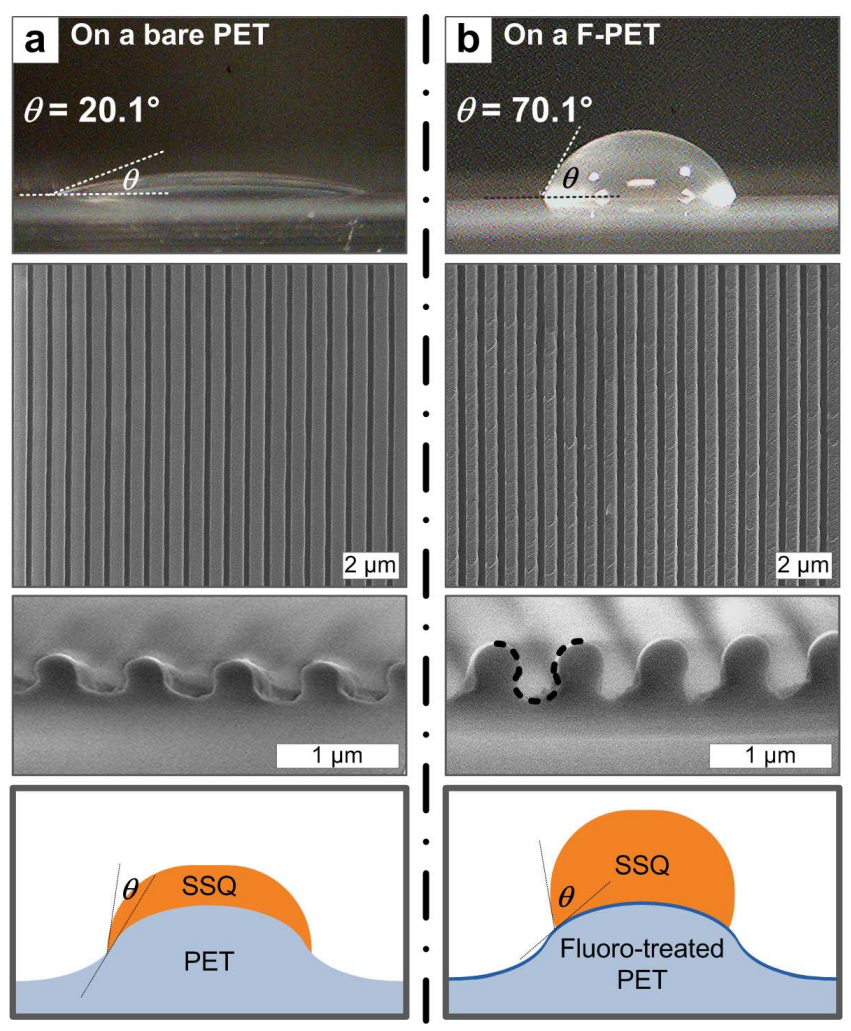

Figure S3. Comparison of nanogratings formed on the surfaces of the same material with different surface properties: (a) normal PET and (b) fluorosilane-treated PET (F-PET). A significant increase in contact angle is observed in a F-PET surface, resulting in the nanograting (processed at $80{ }^{\circ} \mathrm{C}$ ) with higher aspect ratio which is attributed to the improved non-wetting characteristic of the substrate surface that mitigates the reflow of the as-formed liquid resist grating. 


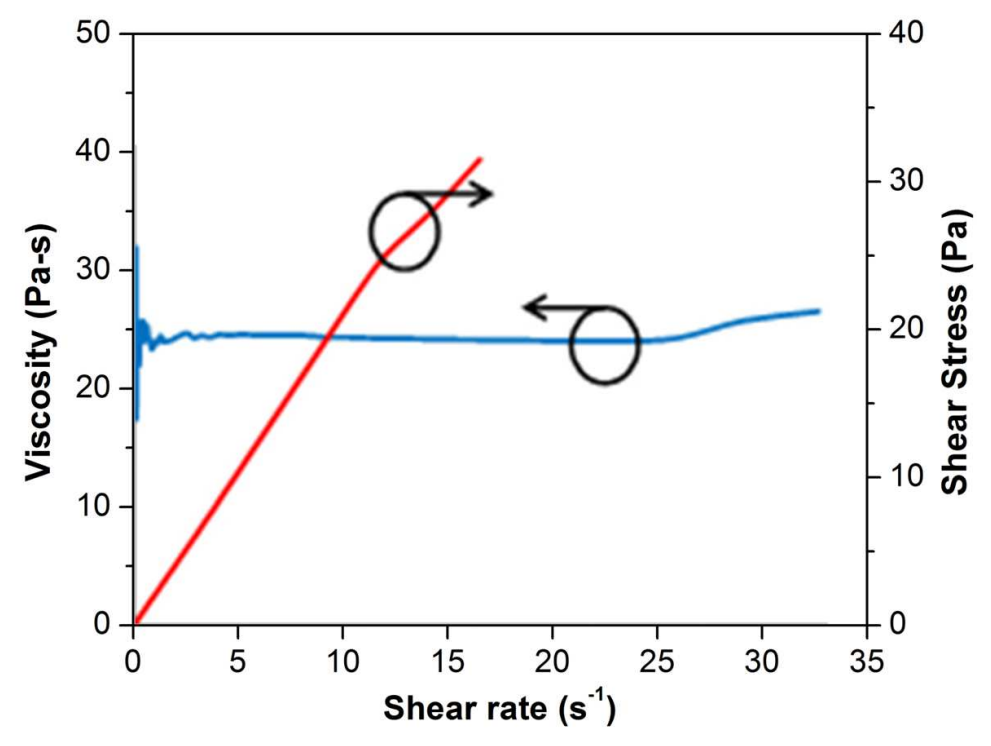

Figure S4. Viscosity and shear stress of liquid SSQ as a function of shear rate. Viscosity remains constant and shear stress increases linearly along the shear rate sweep, indicating that SSQ behaves as a Newtonian fluid. 

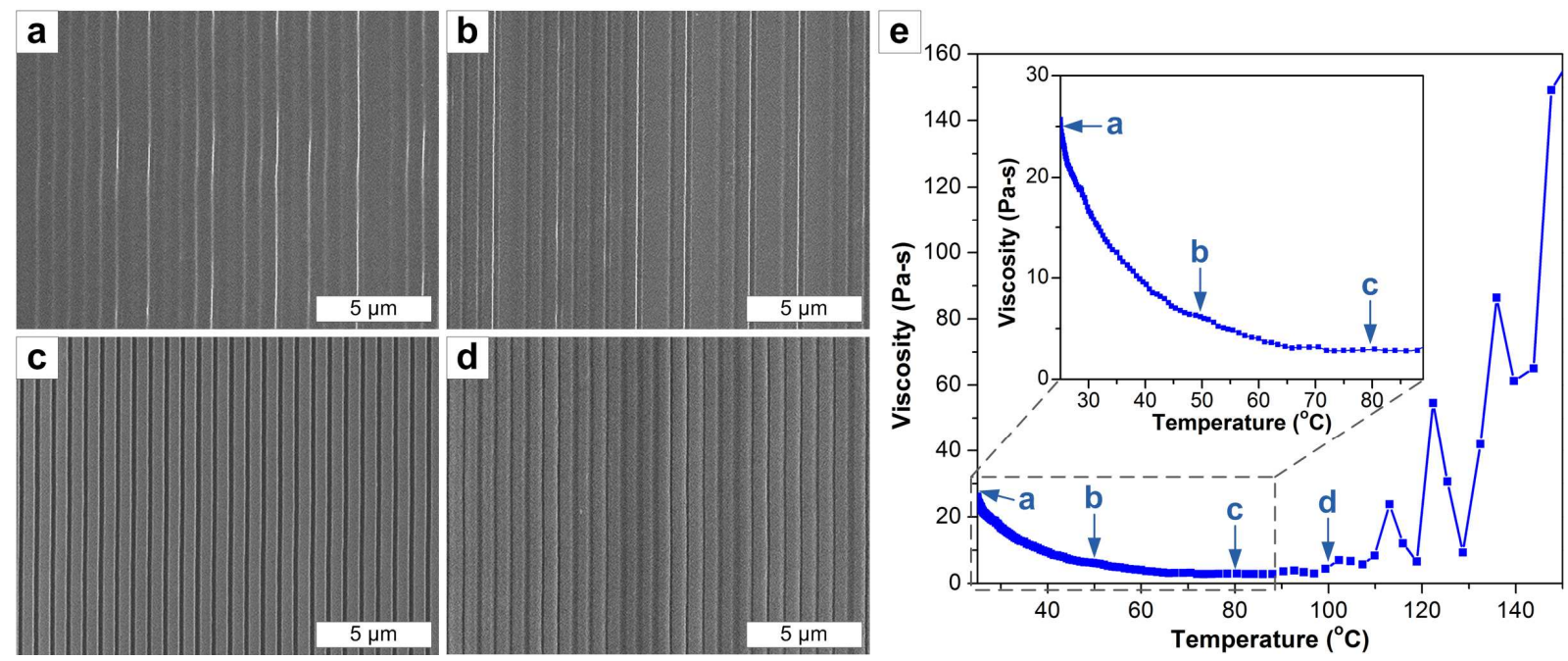

Figure S5. SEM images of $700 \mathrm{~nm}$ period nanogratings formed on the SSQ-coated PET substrates at different processing temperatures: (a) room temperature, (b) $50{ }^{\circ} \mathrm{C}$, (c) $80{ }^{\circ} \mathrm{C}$, and (d) $100{ }^{\circ} \mathrm{C}$. The viscosity of SSQ as a function of temperature is shown in (e), with the marks at which (a)-(d) are processed. The grating depth appears to increase with more faithful profiles from room temperature up to $80{ }^{\circ} \mathrm{C}$, as the SSQ viscosity decreases, while it appears to become shallower when processed at $100{ }^{\circ} \mathrm{C}$ at which the SSQ viscosity increases because of curing effect. The viscosity measurement becomes unstable after $90{ }^{\circ} \mathrm{C}$ presumably due to the 'stick and slip' motions caused by the SSQ curing, but the increasing trend is obvious. 